\title{
Low amounts of mitochondrial reactive oxygen species define human sperm quality
}

\author{
Mónica Marques ${ }^{1,5}$, Ana Paula Sousa ${ }^{1,2}$, Artur Paiva ${ }^{3}$, Teresa Almeida-Santos ${ }^{2,4}$ and \\ João Ramalho-Santos ${ }^{1,5}$ \\ ${ }^{1}$ Biology of Reproduction and Stem Cell Group, CNC-Center for Neuroscience and Cell Biology, University of \\ Coimbra, 3004-517 Coimbra, Portugal, ${ }^{2}$ Human Reproduction Service, University Hospitals of Coimbra, \\ Pr. Mota Pinto, Ed. São Jerónimo, 3000-075 Coimbra, Portugal, ${ }^{3}$ Portuguese Institute for Blood and Transplantation, \\ Coimbra, Portugal, ${ }^{4}$ Faculty of Medicine, University of Coimbra, Coimbra, Portugal and ${ }^{5}$ Department of Life Sciences, \\ University of Coimbra, PO Box 3046, 3001-401 Coimbra, Portugal
}

Correspondence should be addressed to J Ramalho-Santos; Email: jramalho@ci.uc.pt

\begin{abstract}
We have applied the mitochondria-specific superoxide fluorescent probe MitoSOX Red (MitoSOX) to detect mitochondria-specific reactive oxygen species (mROS) production in human sperm samples using flow cytometry. We show that human ejaculates are heterogeneous in terms of mROS production, with three subpopulations clearly detectable, comprising sperm that produce increasing amounts of $\mathbf{m R O S}$ (MitoSOX -, MitoSOX + , and MitoSOX ++ ). The sperm subpopulation producing the lowest amount of $\mathrm{mROS}$ represented the most functional subset of male gametes within the ejaculate, as it was correlated with the highest amount of live and non-apoptotic sperm and increased both in samples with better semen parameters and in samples processed by both density-gradient centrifugation and swim-up, both known to select for higher quality sperm. Importantly, the MitoSOX - subpopulation was clearly more prevalent in samples that gave rise to pregnancies following assisted reproduction. Our work, therefore, not only describe discreet human sperm heterogeneity at the mROS level but also suggests that mROS may represent a strategy to both evaluate sperm samples and isolate the most functional gametes for assisted reproduction.
\end{abstract}

\section{Free Portuguese abstract}

A Portuguese translation of this abstract is freely available at http://www.reproduction-online.org/content/147/6/817/suppl/DC1 Reproduction (2014) 147 817-824

\section{Introduction}

The human ejaculate is a very heterogeneous biological mixture containing subpopulations of sperm with distinct properties and functional abilities (Sousa et al. 2011). Several properties are thought to be needed for a functional mammalian sperm to achieve fertilization, including a morphologically normal structure, motility or the ability to undergo important events such as capacitation and the acrosome reaction, for example (Amaral et al. 2013a). Discrete levels of reactive oxygen species (ROS) have also been proposed as players in the activation of male gamete.

ROS are important in terms of both cell signaling and pathology, and mitochondria-produced ROS (mROS) are especially known to have major roles (reviewed by Sena \& Chandel (2012)). In mammalian sperm, the endogenous production of mROS has been linked with the activity of mitochondrial respiratory chain complexes I and III (Koppers et al. 2008), although the male gamete may have other ROS-producing sources such as NADPH oxidase (Musset et al. 2012). Furthermore, the presence of excessive amounts of leukocytes in the semen seems to be responsible for sperm contact with exogenous (leukocyte-produced) ROS (Henkel 2011). In general, both intracellular and extracellular ROS have been associated not only with sperm damage, such as apoptosis, but also with a positive role in terms of cell functionality (i.e. capacitation; de Lamirande \& Lamothe 2009, Aitken \& Koppers 2011, Tvrda et al. 2011). This difference depends on issues of timing and amount of ROS production.

ROS levels can be correlated with human semen quality, as non-normozoospermic samples seem to have higher amounts than normozoospermic samples (Li et al. 2012), and there is a relationship between poor semen parameters and ROS levels (Wang et al. 2003, Allamaneni et al. 2005, Ramya et al. 2010). However, other studies failed to confirm these findings 
(Zorn et al. 2010). Moreover, there is an association between semen ROS levels and male infertility (Agarwal et al. 2003, 2006) and ROS levels could be correlated with idiopathic male infertility, suggesting a possible diagnostic application (Dohle et al. 2005, Agarwal et al. 2006). Concomitantly, other studies have shown a negative relationship between semen ROS levels and embryo development/pregnancy (Zorn et al. 2003).

Given the heterogeneity of human sperm samples, it would be of interest to determine whether ROS-related properties vary within the population. In fact, mitochondrial function, specifically mitochondrial membrane potential, has been proposed to distinguish better-quality gametes (Sousa et al. 2011). Considering that mitochondria are also the main source of cellular ROS, we thus attempted to identify and characterize distinct human sperm subpopulations based on mROS content and to correlate this data with sperm functionality.

\section{Materials and methods}

\section{Reagents and media}

All reagents were from Sigma-Aldrich and all probes were from Molecular Probes/Invitrogen (Eugene, OR, USA), unless otherwise stated. Several sperm suspension media were used: PBS-glucose-BSA, sperm preparation medium (SPM), simple PBS, and HEPES buffer (10 mM HEPES, $140 \mathrm{mM} \mathrm{NaCl}$, and $\left.2.5 \mathrm{mM} \mathrm{CaCl}_{2}, \mathrm{pH}=7.4\right)$.

SPM (Medicult-Origio, Jyllinge, Denmark) is a commercial medium routinely used for sperm preparation prior to its use in assisted reproductive technologies (ART). PBS-glucose-BSA is PBS supplemented with $0.9 \mathrm{mM} \mathrm{CaCl}_{2}, 5 \mathrm{mM}$ glucose, $0.5 \mathrm{mM}$ $\mathrm{MgCl}_{2}, 3 \%(\mathrm{w} / \mathrm{v}) \mathrm{BSA}$, and 1\% (v/v) Gibco penicillin/streptomycin, $\mathrm{pH}=7.2$ (Amaral et al. 2011).

\section{Sperm sample collection and processing}

Patients undergoing routine semen analysis or fertility treatment were recruited from the Fertility Clinic (University Hospitals of Coimbra, Portugal). All 100 patients signed informed consent forms, and all human material was used in accordance with the appropriate ethical and Internal Review Board (IRB) guidelines provided by the University Hospitals of Coimbra. Fresh semen samples were obtained by masturbation after 3-5 days of sexual abstinence and seminal analysis was carried out in conformity to the World Health Organization Guidelines (WHO 2010). All samples used were analyzed as described by WHO guidelines (WHO 2010), including parameters such as $\mathrm{pH}$, volume, concentration, motility, morphology, and presence or absence of leukocytes. As detailed later on, both samples that had and that lacked leukocytes were used, and analyses were performed on distinct stages of sperm preparation.

In some experiments, the initial ejaculate, or native semen sample (without any conventional treatment), was directly used after two washes with PBS (centrifugation at $800 \boldsymbol{g}$ for $5 \mathrm{~min}$ ) as described (WHO 2010). This pellet was then resuspended in SPM at room temperature for all other experiments. In other assays, native semen samples (without any PBS wash) were further processed by density-gradient centrifugation (DGC), with a 10-min centrifugation at $800 \mathrm{~g}$, using a specific medium (ISolate, Irvine Scientific, Saint Ana, CA, USA) and the isolated fraction was resuspended in SPM. In addition, in some experiments, swim-up was performed following DGC. In this case, the pellet obtained by DGC was gently covered with SPM and incubated at $37^{\circ} \mathrm{C}$ for at least $30 \mathrm{~min}$ to allow motile sperm to spontaneously migrate into the overlaying media, which was then carefully removed, leaving behind non-migrated cells (Amaral et al. 2007, Sousa et al. 2011, Baptista et al. 2013). In experiments with native semen samples we performed a PBS wash only. Regarding the processed samples, for those after DGC the only treatment carried out was DGC; and for those obtained by the swim-up method we performed DGC and swim-up, sequentially (processed samples were not washed with PBS). We used a total of 44 unprocessed samples and 99 processed samples (84 after density-gradient centrifugation and 15 after swim-up). To control for possible differences in sperm viability following different sperm preparation techniques, the eosin/nigrosin assay was used (WHO 2010). Briefly, $10 \mu \mathrm{l}$ sperm suspensions were sequentially mixed with $10 \mu \mathrm{l}$ of $1 \%(\mathrm{w} / \mathrm{v})$ eosin for $30 \mathrm{~s}$ and then with $10 \mu \mathrm{l}$ of $10 \%(\mathrm{w} / \mathrm{v})$ nigrosin for another $30 \mathrm{~s}$. A smear was then prepared and 200 sperm were counted using a bright field microscope and the percentages of non-viable (eosin-stained) and viable sperm were assessed.

\section{Flow cytometry}

For all flow cytometry experiments, a cell suspension with 5 million sperm/ml (final volume of $300 \mu \mathrm{l}$ ) was analyzed using a BD FACS Canto II (Becton Dickinson, Franklin Lakes, NJ, USA) flow cytometer. Fifty thousand cells were acquired in each experimental condition using the FACS DIVA (Becton Dickinson) Software and the results were processed in the Infinicyt V1.5 (Infinicyt, Cytognos, Salamanca, Spain) Software. Control experiments were carried out using cell sorting to ensure that only sperm (and not debris, leucocytes or other round cells; Fig. 1) was being gated and analyzed in all assays, as described previously (Sousa et al. 2011).

To detect mROS, MitoSOX Red (MitoSOX), a specific fluorescent probe for superoxide produced by mitochondria, was used as described previously (Amaral et al. 2013b, Sousa et al. 2013). The MitoSOX Red probe is composed of dihydroethidium, which only reacts with superoxide anion, coupled with and triphenylphosphonium cation that directs the probe to mitochondria. The reaction product exhibits fluorescence after binding DNA (Aitken et al. 2013). All samples were washed with PBS and centrifuged at $800 \mathrm{~g}$ for $5 \mathrm{~min}$. Then, they were incubated with MitoSOX Red at a final concentration of $3 \mu \mathrm{M}$ and in a final volume of $300 \mu \mathrm{l}$ medium (according to different experimental conditions different media were used), for $15 \mathrm{~min}$ at $37^{\circ} \mathrm{C}$ in the dark. At the end of this incubation the cell suspension was washed and analyzed. As a positive control, antimycin A ( $80 \mu \mathrm{M}$ final concentration) was incubated simultaneously with MitoSOX Red. This condition is known to greatly increase the production of mitochondrial ROS (Amaral et al. 2013b). MitoSOX Red fluorescence was 


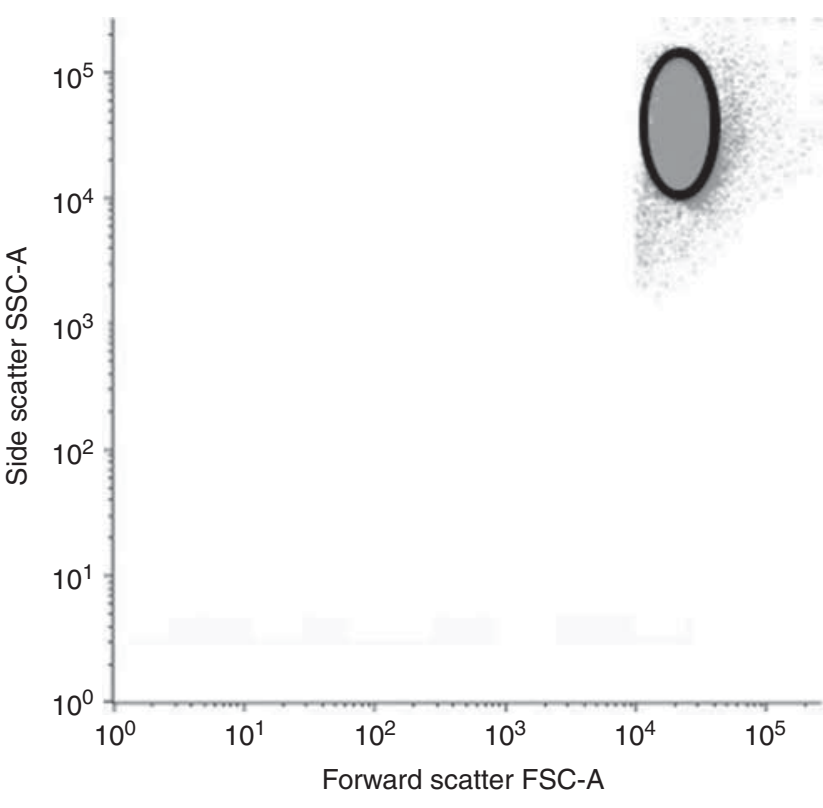

Figure 1 Flow cytometry sperm gating. A representative dot plot obtained to gate sperm using logarithm of side and forward scatter. Round cells such as leukocytes and other debris were excluded.

determined using the blue aragon laser and emitted fluorescence was detected with a bandpass filter of 585/42 nm.

To determine the percentage of viable cells in flow cytometry experiments, the fluorescent dye SYTOX Green was used, as described previously (Varum et al. 2007). This probe stains (nonviable) sperm with a damaged plasma membrane. In this case, $0.1 \mu$ l of SYTOX Green diluted with HEPES buffer was added for each $100 \mu \mathrm{l}$ sperm suspension (final concentration $0.5 \mathrm{nM}$ ). Samples were incubated at room temperature for $15 \mathrm{~min}$ in the dark. Before and after the incubation with the probe, the sample was washed with PBS and centrifuged at $800 \mathrm{~g}$ for $5 \mathrm{~min}$. As a positive control, samples were fixed to induce cell death, using a solution composed of PBS with formaldehyde ( $2 \% \mathrm{v} / \mathrm{v} ; \mathrm{pH}=7.2)$, for $40 \mathrm{~min}$. SYTOX Green fluorescence was detected using a $488 \mathrm{~nm}$ excitation laser to detect the FITC fluorophore, and with a bandpass filter of 530/30 nm.

Sperm apoptosis was assessed using FITC-labeled annexin V (Immunostep, Salamanca, Spain), a calcium-dependent protein with affinity for phosphatidylserine. In intact cells, this probe thus detects the externalization of phosphatidylserine to the outer leaflet of the sperm plasma membrane, a well-known apoptotic event. As previously described (Varum et al. 2007), samples were washed twice with PBS and the pellet was resuspended in HEPES buffer. For each $100 \mu$ l cell suspension, $5 \mu \mathrm{l}$ annexin $\mathrm{V}$ solution were added and the mixture was incubated for $15 \mathrm{~min}$ at room temperature in the dark, as noted in the manufacturer's instructions. Sperm were then washed with HEPES buffer ( $800 \boldsymbol{g}$ for $5 \mathrm{~min}$ ) and the pellet resuspended in HEPES buffer before flow cytometry analysis, using the same settings as described above for SYTOX Green. As a positive control, apoptosis was induced with hydrogen peroxide $(1 \mathrm{mM}$, at $37^{\circ} \mathrm{C}$, for $1 \mathrm{~h}$ ) prior to annexin $\mathrm{V}$ labeling (Varum et al. 2007).

\section{Statistical analysis}

Statistical analysis was performed using the IBM SPSS 20 Software. All variables were checked for normal distribution through the one-sample Kolmogorov-Smirnov test. For two group comparisons, and depending on whether the data had a normal or non-normal distribution, we used either the $t$-test or the Mann-Whitney $U$ non-parametric test respectively. Pearson's coefficient was calculated to correlate different sets of experiments when data had a normal distribution, while Spearman's coefficient was calculated in all other cases. To analyze data related to patients, we used Fisher's exact test. Statistical significance was considered when $P \leq 0.05$. All data are presented as mean \pm s.D.

\section{Results}

\section{MitoSOX subpopulations in human sperm: relationship with apoptotic and viable cells}

In most human sperm samples (both native and prepared), flow cytometry MitoSOX analysis suggested the existence of three clearly distinct subpopulations, a major subpopulation composed of sperm that did not bind the probe (MitoSOX - ), and two with increasing amounts of fluorescence (MitoSOX + and MitoSOX ++ ; Fig. 2A). Of all the samples analyzed, 68\% followed this pattern, while in $14 \%$ only two subpopulations were obvious (MitoSOX - and MitoSOX + ), and in 18\%, a minor MitoSOX +++ subpopulation was detected. As predicted, positive control experiments using antimycin A, a mitochondrial inhibitor of complex III known to cause an increase in mROS, led to an increase in the MitoSOX + subpopulations, with the concomitant disappearance of the MitoSOX - subpopulation (Fig. 2B).

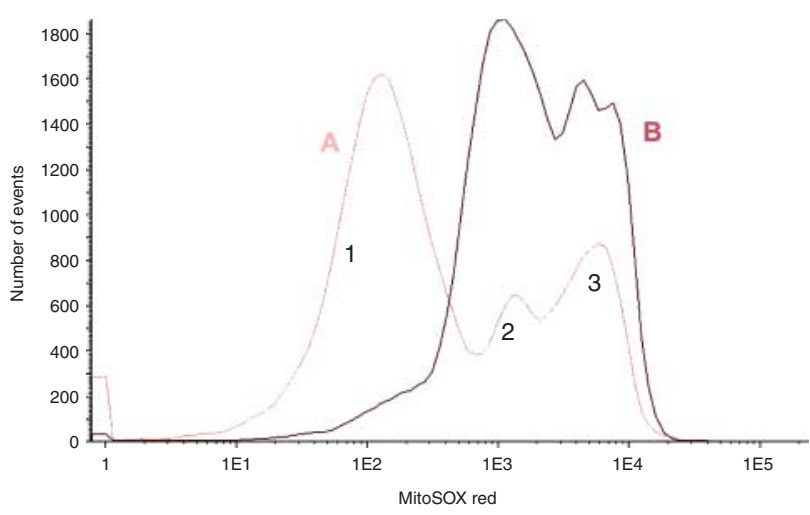

Figure 2 MitoSOX subpopulations in human sperm. Representative examples of histograms obtained after flow cytometry analysis of samples incubated with MitoSOX Red (A) and with MitoSOX Red plus antimycin A (positive control; B). Three different subpopulations are clearly obtained: MitoSOX - (1), MitoSOX + (2), and MitoSOX + + (3). Adding antimycin A results in the disappearance of the MitoSOXsubpopulation, with concomitant increases in the other two subpopulations. 
Table 1 Correlations obtained between sperm subpopulations based on their mROS content and viable and apoptotic cells assayed in the same samples ${ }^{\mathrm{a}}$.

\begin{tabular}{ccc}
\hline MitoSOX - & MitoSOX + & MitoSOX ++ \\
\hline Live cells & & $r=-0.775$ \\
$r=0.758$ & - & $* * * P<0.001$ \\
$* * * P<0.001$ & & $n=23$ \\
$n=23$ & & \\
Apoptotic cells & $r=0.436$ & $r=0.554$ \\
$r=-0.843$ & $* P<0.05$ & $* * P<0.01$ \\
$* * * P<0.001$ & $n=26$ & $n=26$ \\
$n=26$ &
\end{tabular}

${ }^{\mathrm{a} M i t o S O X}$, annexin V (apoptotic sperm), and SYTOX Green (non-viable sperm) assays were carried out by flow cytometry, as described in the 'Materials and methods' section.

When correlating the different amounts of sperm (after DGC) belonging to the distinct MitoSOX subpopulations with the number of apoptotic (annexin V-positive) and viable cells (SYTOX Green-negative) in the same samples, clear relationships were evident (Table 1). Indeed, the presence of MitoSOX - sperm was correlated with both the presence of live and especially of non-apoptotic sperm, while correlations were reversed for the MitoSOX ++ subpopulation and weak or non-existent for the MitoSOX + subpopulation (Table 1). These data seem to suggest that the MitoSOX - subpopulation represents more functional male gametes in a given sample.

\section{MitoSOX subpopulations and leukocytospermia}

In human semen, both the male gamete and leukocytes present in the ejaculate can contribute toward ROS formation. To determine whether the presence of leukocytes in the ejaculate changed sperm ROS production as monitored by MitoSOX, we compared normozoospermic samples (i.e. sperm samples classified as normal using WHO criteria) containing large quantities of leukocytes (leukocytospermia, $n=30$ ) with normozoospermic samples lacking leukocytes $(n=61)$. Importantly, as stated in the Materials and methods' section, only sperm were gated and analyzed in all these experiments, and no differences were found between samples with or without leukocytes, i.e. the presence of leukocytes in the ejaculate did not change the number of sperm in the three distinct MitoSOX subpopulations detected ( $P>0.05$, data not shown). This was true whether the initial native semen samples were considered, or if samples were analyzed after densitygradient centrifugation (DGC; data not shown).

\section{MitoSOX subpopulations in human sperm samples with distinct initial quality}

To determine whether MitoSOX subpopulations are related to distinct sperm quality parameters, we first decided to determine whether there were differences between normozoospermic samples and samples that would not be classified as normal using WHO criteria. Interestingly, normozoospermic and non-normozoospermic semen samples were not statistically distinct if the initial unprocessed semen samples were considered (Fig. 3A), but normozoospermic samples had a higher percentage of the MitoSOX - subpopulation (and a lower percentage of the MitoSOX ++ subpopulation) after sperm processing for ART (Fig. 3B).

\section{MitoSOX subpopulations in native and prepared human sperm samples}

To further determine the possible relevance of MitoSOX subpopulations within normal samples, we evaluated their prevalence in both native normozoospermic semen samples, and in the same samples following processing by DGC and swim-up, as usually carried out to isolate more functional male gametes for ART. As shown (Fig. 4). The MitoSOX - subpopulation was very significantly increased following both sperm preparation techniques, at the expense of either the MitoSOX ++ (DGC) or MitoSOX + (swim-up) subpopulations.
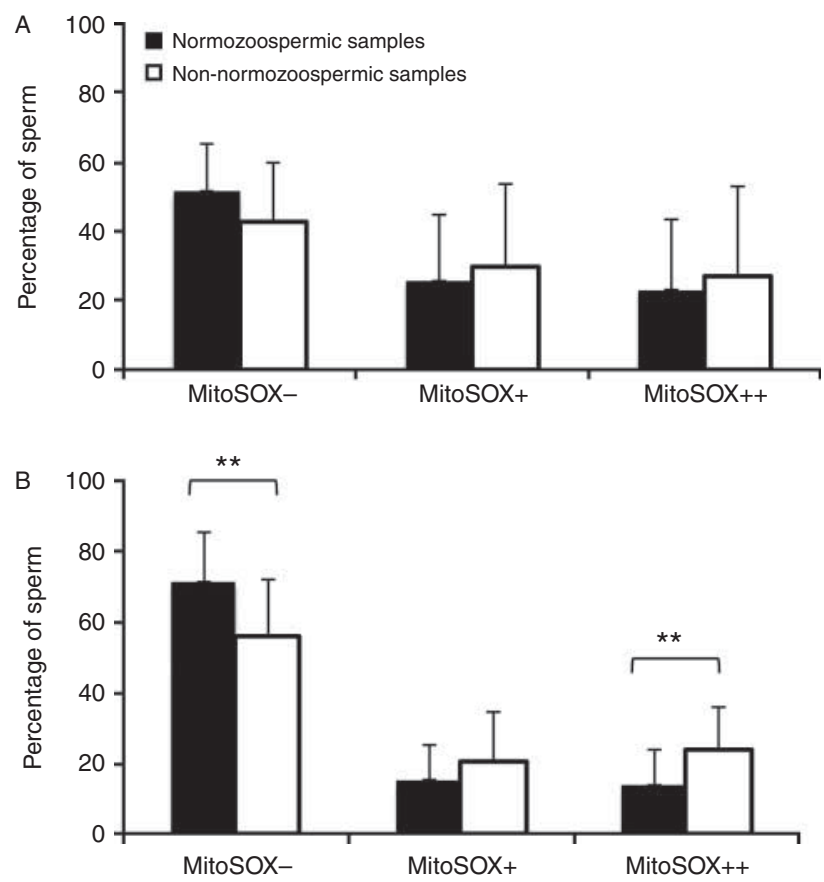

Figure 3 MitoSOX subpopulations in normozoospermic and nonnormozoospermic human sperm samples. Sperm subpopulations (MitoSOX -, MitoSOX + , and MitoSOX ++ ) were quantified in both normozoospermic $(n=61)$ and non-normozoospermic $(n=20)$ human sperm samples, both in native semen samples (A) one-sample Kolmogorov-Smirnov test and $t$-test were performed, and in samples following processing by density-gradient centrifugation (DGC) (B) onesample Kolmogorov-Smirnov test and $t$-test were performed. Data are presented as mean \pm s.D. ${ }^{* *} P \leq 0.01$. 

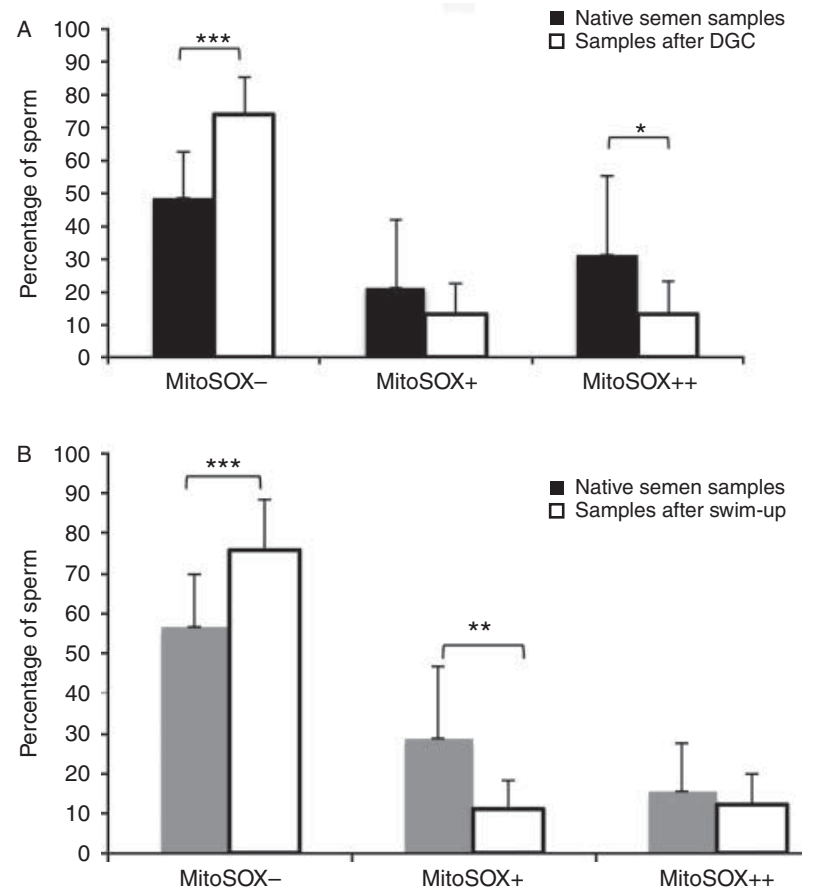

Figure 4 MitoSOX subpopulations in native and processed human sperm. The three different sperm subpopulations previously identified according to MitoSOX reactivity (MitoSOX -, MitoSOX +, and MitoSOX ++ ) were quantified in native semen samples, and in those same samples following processing by (A) density-gradient centrifugation (DGC, $n=13$; one-sample Kolmogorov-Smirnov test and $t$-test were performed) and (B) swim-up ( $n=15$; one-sample KolmogorovSmirnov test and $t$-test were performed). Data are presented as mean \pm S.D. ${ }^{*} P \leq 0.05,{ }^{* *} P \leq 0.01$, and ${ }^{* *} P \leq 0.001$.

\section{Correlation of pregnancy with sperm MitoSOX subpopulations}

Finally, we attempted to determine whether the different MitoSOX subpopulations might be correlated with ART success, and thus with the achievement of pregnancy. As shown in Fig. 5, a positive immunological pregnancy test $(I P T+)$ result was significantly associated with a higher MitoSOX - subpopulation, and with lower amounts of MitoSOX ++ subpopulation. Patient data are presented in Table 2, and it is noteworthy that the pregnant and non-pregnant groups were otherwise statistically indistinguishable when clinically relevant parameters were considered. We considered a total of 26 patients for which the infertility cause could be female, male, both, or idiopathic. In our population, the most prominent female infertility cause was tubal factor and, after the female factor, idiopathic infertility is the most common cause listed. In these patients, IVF was performed more often than ICSI, but this is true for both IPT - and IPT + . In our population one to three embryos were transferred, with an average of two embryos for IPT+ and 1.88 for IPT - . For all the parameters described above, we found no statistically significant differences between populations. We also checked whether there was any difference according to the age of the patient (female or male) that could be related to pregnancy success, and again this was not the case (Table 2).

\section{Discussion}

Both pathological and physiological effects have been described for ROS in sperm. In this study, the goal was to determine whether endogenous ROS levels, and particularly mROS, could identify sperm subpopulations with distinct characteristics.

We determined that most human sperm samples comprised three subpopulations, as defined using the fluorescent probe for mROS MitoSOX Red and flow cytometry analysis. When simultaneously considering apoptotic markers and viability, the MitoSOX - subpopulation (sperm that showed little or no probe staining) included better-quality sperm, while the increase in mROS levels in the MitoSOX + and MitoSOX ++ subpopulations resulted in progressively worse sperm characteristics. The relationship between sperm mROS levels and apoptosis is known (Koppers et al. 2011, Aitken et al. 2012a), as is the connection between ROS levels and sperm viability (Marchetti et al. 2002, Espinoza et al. 2009). Furthermore, the link between mROS production and apoptosis is recognized in other cell types (Sinha et al. 2013), although the particular compartmentalization of sperm cell might result in different types of cellular damage (Aitken et al. 2012b).

The presence of leukocytes in a semen sample has been correlated with sperm damage, and this is thought to be caused by ROS production in the white blood cells

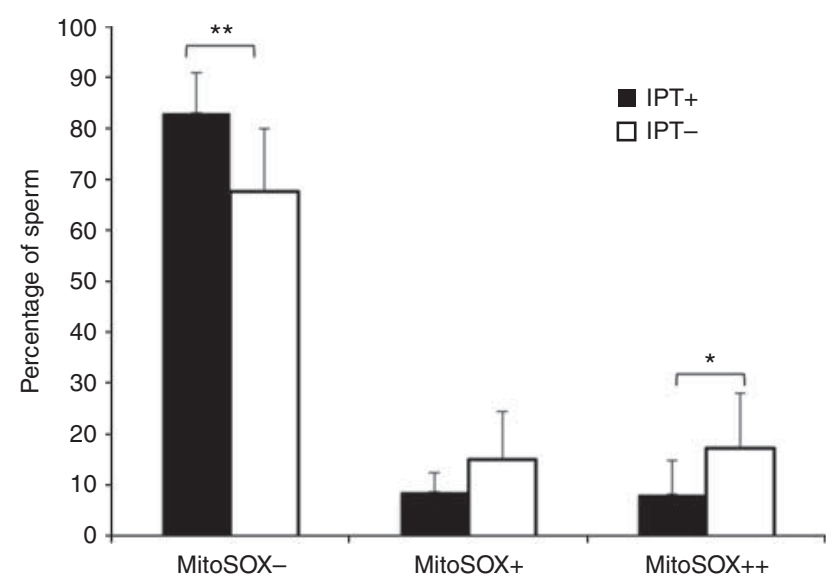

Figure 5 MitoSOX subpopulations in sperm samples and pregnancy results. Sperm subpopulations (MitoSOX -, MitoSOX+, and MitoSOX ++ ) were quantified in human sperm samples used in assisted reproduction technologies (ART) that gave rise to a positive immunological pregnancy test (IPT,$+ n=9)$ and that did not result in pregnancy (IPT,$- n=17)$. Data are presented as mean \pm s.D. ${ }^{*} P \leq 0.05$ and ${ }^{* *} P \leq 0.01$. One-sample Kolmogorov-Smirnov test and $t$-test were performed. 
Table 2 Pregnancy data for samples used in this study: patient age, number of embryos transferred (mean \pm s.D.), cause of infertility, primary or secondary sterility and technique performed (number of cases).

\begin{tabular}{|c|c|c|c|}
\hline & IPT + & IPT - & $P$ value \\
\hline $\begin{array}{l}\text { Number of } \\
\text { patients }\end{array}$ & 9 & 17 & - \\
\hline \multicolumn{4}{|l|}{ Age } \\
\hline Female & $35.22 \pm 3.23$ & $35.23 \pm 2.84$ & 0.922 \\
\hline Male & $38.66 \pm 5.36$ & $35.58 \pm 3.82$ & 0.102 \\
\hline $\begin{array}{l}\text { Number of trans- } \\
\text { ferred embryo }\end{array}$ & $2 \pm 0.60$ & $1.88 \pm 0.70$ & 0.659 \\
\hline Infertility cause & & - & 0.574 \\
\hline Male factor & 1 & 2 & - \\
\hline Female factor & 2 & 8 & - \\
\hline $\begin{array}{l}\text { Male and female } \\
\text { factor }\end{array}$ & 1 & 2 & - \\
\hline Idiopathic & 5 & 5 & - \\
\hline Type of infertility & & & 0.357 \\
\hline Primary & 1 & 11 & - \\
\hline Secondary & 8 & 6 & - \\
\hline ART type & & & 0.664 \\
\hline IVF & 7 & 11 & - \\
\hline ICSI & 2 & 6 & - \\
\hline
\end{tabular}

IPT +, immunological pregnancy test positive; IPT - , immunological pregnancy test negative; ART, assisted reproductive technique.

(Henkel 2011). Although some groups reported that there is a relationship between endogenous sperm ROS levels and an excess of leukocytes (Henkel et al. 2005, Mupfiga et al. 2013), we found no difference between leukocytospermic samples and non-leukocytospermic samples regarding mROS levels both in the native sample and after density gradient centrifugation, as also reported by others (Henkel et al. 2010), suggesting that the presence of leukocytes in the semen does not seem to influence endogenous sperm production of mROS.

In terms of classic sperm parameters (using WHO standards), non-normozoospermic samples had a lower percentage of sperm in the MitoSOX - subpopulation than normozoospermic samples, if analysis was carried out following density gradient centrifugation. Conversely, the MitoSOX ++ subpopulation was increased in non-normozoospermic samples. Other groups have found relationships between ROS levels and sperm quality or alterations in sperm parameters, including motility, morphology, and concentration (Wang et al. 2003, Henkel et al. 2005, Cocuzza et al. 2008, Aitken et al. 2012c, Li et al. 2012, Ferramosca et al. 2013). Conventional processing techniques used to prepare sperm for ART are often associated with an induction of sperm ROS, likely due to both the centrifugation itself and the removal of putative semen antioxidants (Agarwal et al. 1994a, Thilagavathi et al. 2012). We here show that prepared samples had a higher percentage of sperm in the MitoSOX - subpopulation and a lower percentage of MitoSOX ++ sperm. These differences, both between normozoospermic and non-normozoospermic samples, and samples before and after centrifugation, might result from the removal of dead cells (Larson et al. 1999).
Indeed, others have also described a decrease in superoxide anion using this procedure, although an increase in hydrogen peroxide levels was also found (Mahfouz et al. 2010).

On the other hand, when comparing native sample with the migrated subpopulation obtained after swim-up, which we have previously shown to be beneficial to isolate better-quality sperm in terms of capacitation and chromatin integrity (Sousa et al. 2011, Tavares et al. 2013), we also detect a higher percentage of sperm in the MitoSOX - subpopulation relative to the native sample. Interestingly, with this methodology the subpopulation affected in the opposite direction was the MitoSOX + subpopulation, which tells us that the swim-up method is suitable for more subtly isolating a subpopulation with lower ROS, given that the MitoSOX ++ subpopulation is already greatly decreased prior to performing swim-up separation (Agarwal et al. 1994b). Migrated samples also predictably had higher viability than native samples, although there was no statistically significant difference in terms of apoptosis.

Finally, sperm ROS levels have been suggested to play a role in infertility (Pasqualotto et al. 2001, Agarwal et al. 2003, Venkatesh et al. 2011), and we obtained statistically significant differences between samples that did or did not give rise to pregnancy following ART in terms of mROS. In agreement with the data discussed so far, successful outcomes could be linked to samples with a higher percentage of sperm with lower mROS. Although others have reported no difference in ROS levels between samples that give rise to pregnancy and samples that did not, but differences were found if a pre-established ROS level cut-off value was disregarded (Zorn et al. 2003). To exclude other parameters that could be related to pregnancy success, we also evaluated male and female age, the type and cause of infertility, the technique performed, and the number of embryos transferred. We did not find any association between these parameters and pregnancy results, suggesting that in this case of the parameters monitored only sperm mROS are influencing pregnancy.

In summary, we show that human sperm samples are heterogeneous in terms of mROS generated and can be divided into discreet subpopulations. Moreover, the subpopulation with lower amounts of mROS (MitoSOX -) consistently seemed to include better-quality sperm, whatever the criteria used, from functional markers to issues related to sample preparation and ultimately including the establishment of pregnancy following ART. Given that ROS are known to play a role in sperm function, it seems odd that the subpopulation with better quality sperm is the one negative for mROS. However, it is possible that the chosen marker is not sensitive enough to detect basal levels of mitochondrial superoxide required or that basal ROS are obtained via other sources, such as NADPH oxidase, with increasing levels of mROS (as monitored with this particular 
probe/assay) representing the pathological amounts known to adversely affect sperm function. More studies with a larger patient cohort and distinct methodologies are required to clarify the role of ROS in sperm (both mitochondrial and non-mitochondrial, both endogenous and exogenous), for example determining whether distinct populations are related to distinct levels of sperm capacitation. The establishment of possible cut-off values that may allow for the distinction between basal physiological levels from pathological levels might also be possible. Regardless, this study shows that mROS can identify a better-quality sperm subpopulation within a human ejaculate and that the endogenous mROS levels are related to sperm quality and ART outcomes.

\section{Declaration of interest}

The authors declare that there is no conflict of interest that could be perceived as prejudicing the impartiality of the research reported.

\section{Funding}

Center for Neuroscience and Cell Biology (CNC) funding is supported by Fundação para a Ciência e Tecnologia (FCT) (PEst-C/SAU/LA0001/2011).

\section{Acknowledgements}

All laboratory members are thanked for helpful discussions, and the Center for Neuroscience and Cell Biology (CNC) statistical personnel is thanked for assistance. This work was done as part of the Masters Program in Cell and Molecular Biology at the Department of Life Sciences, University of Coimbra (M Marques). CNC funding is supported by FCT (PEst-C/SAU/LA0001/2011).

\section{References}

Agarwal A, Ikemoto I \& Loughlin KR 1994a Effect of sperm washing on levels of reactive oxygen species in semen. Archives of Andrology $\mathbf{3 3}$ 157-162. (doi:10.3109/01485019408987819)

Agarwal A, Ikemoto I \& Loughlin KR 1994b Levels of reactive oxygen species before and after sperm preparation - comparison of swim-up and L4 Filtration. Archives of Andrology 32 169-174. (doi:10.3109/ 01485019408987783)

Agarwal A, Saleh RA \& Bedaiwy MA 2003 Role of reactive oxygen species in the pathophysiology of human reproduction. Fertility and Sterility $\mathbf{7 9}$ 829-843. (doi:10.1016/S0015-0282(02)04948-8)

Agarwal A, Sharma RK, Nallella KP, Thomas AJ, Alvarez JG \& Sikka SC 2006 Reactive oxygen species as an independent marker of male factor infertility. Fertility and Sterility 86 878-885. (doi:10.1016/j.fertnstert. 2006.02.111)

Aitken RJ \& Koppers AJ 2011 Apoptosis and damage in human spermatozoa. Asian Journal of Andrology 13 36-42. (doi:10.1038/aja.2010.68)

Aitken RJ, Whiting S, De Iuliis GN, McClymont S, Mitchell LA \& Baker MA 2012a Electrophilic aldehydes generated by sperm metabolism activate mitochondrial reactive oxygen species generation and apoptosis by targeting succinate dehydrogenase. Journal of Biological Chemistry 287 33048-33060. (doi:10.1074/jbc.M112.366690)
Aitken RJ, Jones KT \& Robertson SA $2012 b$ Reactive oxygen species and sperm function - in sickness and in health. Journal of Andrology 33 1096-1106. (doi:10.2164/jandrol.112.016535)

Aitken RJ, Gibb Z, Mitchell LA, Lambourne SR, Connaughton HS \& De Iuliis GN 2012c Sperm motility is lost in vitro as a consequence of mitochondrial free radical production and the generation of electrophilic aldehydes but can be significantly rescued by the presence of nucleophilic thiols. Biology of Reproduction 87 (5) 110. (doi:10.1095/ biolreprod.112.102020)

Aitken RJ, Smith TB, Lord T, Kuczera L, Koppers AJ, Naumovski N, Connaughton H, Baker MA \& De Iuliis GN 2013 On methods for the detection of reactive oxygen species generation by human spermatozoa: analysis of the cellular responses to catechol oestrogen, lipid aldehyde, menadione and arachidonic acid. Andrology 1 192-205. (doi:10.1111/ j.2047-2927.2012.00056.x)

Allamaneni SS, Agarwal A, Nallella KP, Sharma RK, Thomas AJ Jr \& Sikka SC 2005 Characterization of oxidative stress status by evaluation of reactive oxygen species levels in whole semen and isolated spermatozoa. Fertility and Sterility 83 800-803. (doi:10.1016/j.fertnstert.2004.05.106)

Amaral A, Ramalho-Santos J \& St John JC 2007 The expression of polymerase gamma and mitochondrial transcription factor $\mathrm{A}$ and the regulation of mitochondrial DNA content in mature human sperm. Human Reproduction 22 1585-1596. (doi:10.1093/humrep/dem030)

Amaral A, Paiva C, Baptista M, Sousa AP \& Ramalho-Santos J 2011 Exogenous glucose improves long-standing human sperm motility, viability, and mitochondrial function. Fertility and Sterility 96 848-850. (doi:10.1016/j.fertnstert.2011.07.1091)

Amaral A, Lourenco B, Marques M \& Ramalho-Santos J 2013a Mitochondria functionality and sperm quality. Reproduction $\mathbf{1 4 6}$ R163-R174. (doi:10.1530/REP-13-0178)

Amaral S, Redmann K, Sanchez V, Mallidis C, Ramalho-Santos J \& Schlatt S $2013 b$ UVB irradiation as a tool to assess ROS-induced damage in human spermatozoa. Andrology 1 707-714. (doi:10.1111/j.2047-2927. 2013.00098.x)

Baptista M, Publicover SJ \& Ramalho-Santos J 2013 In vitro effects of cationic compounds on functional human sperm parameters. Fertility and Sterility 99 705-712. (doi:10.1016/j.fertnstert.2012.11.008)

Cocuzza M, Athayde KS, Agarwal A, Sharma R, Pagani R, Lucon AM, Srougi M \& Hallak J 2008 Age-related increase of reactive oxygen species in neat semen in healthy fertile men. Urology 71 490-494. (doi:10.1016/j.urology.2007.11.041)

Dohle GR, Colpi GM, Hargreave TB, Papp GK, Jungwirth A, Weidner W \& Infertility EWGM 2005 EAU guidelines on male infertility. European Urology 48 703-711. (doi:10.1016/j.eururo.2005.06.002)

Espinoza JA, Schulz MA, Sanchez R \& Villegas JV 2009 Integrity of mitochondrial membrane potential reflects human sperm quality. Andrologia 41 51-54. (doi:10.1111/j.1439-0272.2008.00878.x)

Ferramosca A, Provenzano SP, Montagna DD, Coppola L \& Zara V 2013 Oxidative stress negatively affects human sperm mitochondrial respiration. Urology 82 78-83. (doi:10.1016/j.urology.2013.03.058)

Henkel RR 2011 Leukocytes and oxidative stress: dilemma for sperm function and male fertility. Asian Journal of Andrology 13 43-52. (doi:10. 1038/aja.2010.76)

Henkel R, Kierspel E, Stalf T, Mehnert C, Menkveld R, Tinneberg HR, Schill WB \& Kruger TF 2005 Effect of reactive oxygen species produced by spermatozoa and leukocytes on sperm functions in non-leukocytospermic patients. Fertility and Sterility 83 635-642. (doi:10.1016/ j.fertnstert.2004.11.022)

Henkel R, Bastiaan HS, Schuller S, Hoppe I, Starker W \& Menkveld R 2010 Leucocytes and intrinsic ROS production may be factors compromising sperm chromatin condensation status. Andrologia 42 69-75. (doi:10.1111/j.1439-0272.2009.00967.x)

Koppers AJ, De luliis GN, Finnie JM, McLaughlin EA \& Aitken RJ 2008 Significance of mitochondrial reactive oxygen species in the generation of oxidative stress in spermatozoa. Journal of Clinical Endocrinology and Metabolism 93 3199-3207. (doi:10.1210/jc.2007-2616)

Koppers AJ, Mitchell LA, Wang P, Lin M \& Aitken RJ 2011 Phosphoinositide 3-kinase signalling pathway involvement in a truncated apoptotic cascade associated with motility loss and oxidative DNA damage in human spermatozoa. Biochemical Journal 436 687-698. (doi:10.1042/ BJ20110114) 
de Lamirande E \& Lamothe G 2009 Reactive oxygen-induced reactive oxygen formation during human sperm capacitation. Free Radical Biology \& Medicine 46 502-510. (doi:10.1016/j.freeradbiomed.2008. 11.004)

Larson KL, Brannian JD, Timm BK, Jost LK \& Evenson DP 1999 Density gradient centrifugation and glass wool filtration of semen remove spermatozoa with damaged chromatin structure. Human Reproduction 14 2015-2019. (doi:10.1093/humrep/14.8.2015)

Li Z, Zhou Y, Liu R, Lin H, Liu W, Xiao W \& Lin Q 2012 Effects of semen processing on the generation of reactive oxygen species and mitochondrial membrane potential of human spermatozoa. Andrologia 44 157-163. (doi:10.1111/j.1439-0272.2010.01123.x)

Mahfouz RZ, du Plessis SS, Aziz N, Sharma R, Sabanegh E \& Agarwal A 2010 Sperm viability, apoptosis, and intracellular reactive oxygen species levels in human spermatozoa before and after induction of oxidative stress. Fertility and Sterility 93 814-821. (doi:10.1016/ j.fertnstert.2008.10.068)

Marchetti C, Obert G, Deffosez A, Formstecher P \& Marchetti P 2002 Study of mitochondrial membrane potential, reactive oxygen species, DNA fragmentation and cell viability by flow cytometry in human sperm. Human Reproduction 17 1257-1265. (doi:10.1093/humrep/17.5.1257)

Mupfiga C, Fisher D, Kruger T \& Henkel R 2013 The relationship between seminal leukocytes, oxidative status in the ejaculate, and apoptotic markers in human spermatozoa. Systems Biology in Reproductive Medicine 59 304-311. (doi:10.3109/19396368.2013.821540)

Musset B, Clark RA, DeCoursey TE, Petheo GL, Geiszt M, Chen Y, Cornell JE, Eddy CA, Brzyski RG \& El Jamali A 2012 NOX5 in human spermatozoa: expression, function, and regulation. Journal of Biological Chemistry 287 9376-9388. (doi:10.1074/jbc.M111.314955)

Pasqualotto FF, Sharma RK, Kobayashi H, Nelson DR, Thomas AJ Jr \& Agarwal A 2001 Oxidative stress in normospermic men undergoing infertility evaluation. Journal of Andrology 22 316-322. (doi:10.1002/ j.1939-4640.2001.tb02185.x)

Ramya T, Misro MM, Sinha D \& Nandan D 2010 Sperm function and seminal oxidative stress as tools to identify sperm pathologies in infertile men. Fertility and Sterility 93 297-300. (doi:10.1016/j.fertnstert.2009. 05.074)

Sena LA \& Chandel NS 2012 Physiological roles of mitochondrial reactive oxygen species. Molecular Cell 48 158-167. (doi:10.1016/j.molcel. 2012.09.025)

Sinha K, Das J, Pal PB \& Sil PC 2013 Oxidative stress: the mitochondriadependent and mitochondria-independent pathways of apoptosis. Archives of Toxicology 87 1157-1180. (doi:10.1007/s00204-0131034-4)

Sousa AP, Amaral A, Baptista M, Tavares R, Caballero Campo P, Caballero Peregrin P, Freitas A, Paiva A, Almeida-Santos T \& Ramalho-Santos J 2011 Not all sperm are equal: functional mitochondria characterize a subpopulation of human sperm with better fertilization potential. PLOS ONE 6 e18112. (doi:10.1371/journal.pone.0018112)
Sousa MI, Amaral S, Tavares RS, Paiva C \& Ramalho-Santos J 2013 Concentration-dependent Sildenafil citrate (Viagra) effects on ROS production, energy status, and human sperm function. Systems Biology in Reproductive Medicine 60 (2) 72-79.

Tavares RS, Silva AF, Lourenco B, Almeida-Santos T, Sousa AP \& Ramalho-Santos J 2013 Evaluation of human sperm chromatin status after selection using a modified Diff-Quik stain indicates embryo quality and pregnancy outcomes following in vitro fertilization. Andrology 1 830-837. (doi:10.1111/j.2047-2927.2013.00127.x)

Thilagavathi J, Venkatesh S, Kumar R \& Dada R 2012 Segregation of sperm subpopulations in normozoospermic infertile men. Systems Biology in Reproductive Medicine 58 313-318. (doi:10.3109/19396368.2012. 706361)

Tvrda E, Knazicka Z, Bardos L, Massanyi P \& Lukac N 2011 Impact of oxidative stress on male fertility - a review. Acta Veterinaria Hungarica 59 465-484. (doi:10.1556/AVet.2011.034)

Varum S, Bento C, Sousa AP, Gomes-Santos CS, Henriques P, AlmeidaSantos T, Teodosio C, Paiva A \& Ramalho-Santos J 2007 Characterization of human sperm populations using conventional parameters, surface ubiquitination, and apoptotic markers. Fertility and Sterility 87 572-583. (doi:10.1016/j.fertnstert.2006.07.1528)

Venkatesh S, Shamsi MB, Dudeja S, Kumar R \& Dada R 2011 Reactive oxygen species measurement in neat and washed semen: comparative analysis and its significance in male infertility assessment. Archives of Gynecology and Obstetrics 283 121-126. (doi:10.1007/s00404-010-1645-4)

Wang X, Sharma RK, Gupta A, George V, Thomas AJ, Falcone T \& Agarwal A 2003 Alterations in mitochondria membrane potential and oxidative stress in infertile men: a prospective observational study. Fertility and Sterility 80 (Suppl 2) 844-850. (doi:10.1016/S00150282(03)00983-X)

WHO 2010 In WHO Laboratory Manual for the Examination and Processing of Human Semen. 5th ed. Geneva, Switzerland: World Health Organization Press.

Zorn B, Vidmar G \& Meden-Vrtovec H 2003 Seminal reactive oxygen species as predictors of fertilization, embryo quality and pregnancy rates after conventional in vitro fertilization and intracytoplasmic sperm injection. International Journal of Andrology 26 279-285. (doi:10.1046/ j.1365-2605.2003.00424.x)

Zorn B, Ihan A, Kopitar AN, Kolbezen M, Sesek-Briski A \& MedenVrtovec H 2010 Changes in sperm apoptotic markers as related to seminal leukocytes and elastase. Reproductive Biomedicine Online 21 84-92. (doi:10.1016/j.rbmo.2010.03.016)

Received 12 December 2013

First decision 23 December 2013

Revised manuscript received 6 February 2014

Accepted 17 February 2014 